Dementia

and Geriatric

Cognitive Disorders
Dement Geriatr Cogn Disord 2014;38:375-388

DOI: 10.1159/000365548

Acceprea: June 20, ZUI4

Published online: September 5, 2014
C 2014 S. Karger AG, Basel

$1420-8008 / 14 / 0386-0375 \$ 39.50 / 0$

www.karger.com/dem

\title{
Patterns of Hippocampal Tau Pathology Differentiate Neurodegenerative Dementias
}

\author{
Ivan Milenkovic $^{a}$ Tatjana Petrov ${ }^{b}$ Gabor G. Kovacs ${ }^{a}$ \\ ${ }^{a}$ Institute of Neurology, Medical University of Vienna, Vienna, and ${ }^{\mathrm{b}}$ Institute of Science and \\ Technology, Klosterneuburg, Austria
}

\section{Key Words}

Hippocampus · Tauopathies · Dementia - Selective vulnerability

\begin{abstract}
Background/Aims: Deposits of phosphorylated tau protein and convergence of pathology in the hippocampus are the hallmarks of neurodegenerative tauopathies. Thus we aimed to evaluate whether regional and cellular vulnerability patterns in the hippocampus distinguish tauopathies or are influenced by their concomitant presence. Methods: We created a heat map of phospho-tau (AT8) immunoreactivity patterns in 24 hippocampal subregions/layers in individuals with Alzheimer's disease (AD)-related neurofibrillary degeneration $(n=40)$, Pick's disease $(n=8)$, progressive supranuclear palsy $(n=7)$, corticobasal degeneration $(n=$ 6), argyrophilic grain disease (AGD, $n=18)$, globular glial tauopathy $(n=5)$, and tau-astrogliopathy of the elderly $(n=10)$. AT8 immunoreactivity patterns were compared by mathematical analysis. Results: Our study reveals disease-specific hot spots and regional selective vulnerability for these disorders. The pattern of hippocampal AD-related tau pathology is strongly influenced by concomitant AGD. Mathematical analysis reveals that hippocampal involvement in primary tauopathies is distinguishable from early-stage AD-related neurofibrillary degeneration. Conclusion: Our data demonstrate disease-specific AT8 immunoreactivity patterns and hot spots in the hippocampus even in tauopathies, which primarily do not affect the hippocampus. These hot spots can be shifted to other regions by the co-occurrence of tauopathies like AGD. Our observations support the notion that globular glial tauopathies and tau-astrogliopathy of the elderly are distinct entities.


Milenkovic et al.: Patterns of Hippocampal Tau Pathology Differentiate

Neurodegenerative Dementias

\section{Introduction}

Progressive loss of neurons and intra- and extracellular deposition of proteins constitute the major neuropathologic features of neurodegenerative diseases [1]. The most important proteins include the microtubule-associated protein tau, prion protein, amyloid- $\beta, \alpha$-synuclein, fused in sarcoma, and TAR DNA-binding protein 43 [1]. Deposition of the abnormally phosphorylated tau is a hallmark of a group of neurodegenerative diseases designated as tauopathies. These include Alzheimer's disease (AD), argyrophilic grain disease (AGD), corticobasal degeneration (CBD), progressive supranuclear palsy (PSP), Pick's disease (PiD) [2], and neurofibrillary tangle-only dementia [3], as well as disorders with globular glial inclusions, also called globular glial tauopathies (GGTs) [4]. In addition, there are constellations of tauopathies seen in elderly patients, which cannot be diagnosed by established neuropathologic diagnostic criteria, characterized by tau-astrogliopathy as a common feature [5, 6]. Depending on the isoform of the deposited tau protein, tauopathies are grouped as either 3-repeat (R)-predominant (e.g., PiD), 4R-predominant (e.g., AGD, CBD, PSP, GGT, and tau-astrogliopathy of the elderly) or mixed 3R/4R (e.g., AD and neurofibrillary tangle dementia) [7-9].

The diversity of phenotypes associated with tauopathies is related to the deposition of pathologic tau protein in distinct brain areas and in distinct cellular populations $[10,11]$. The anatomical distribution (i.e. subcortical, allocortical or isocortical) of tau pathology considerably varies between tauopathies. However, tau pathology converges in the hippocampus and entorhinal cortex (EC) in all of them. The hippocampal formation comprises the dentate gyrus (DG), hippocampus and subiculum [12], and is essential for navigation [13] and different memory functions, including spatial [14-18] and declarative memory [19, 20]. Mostly unidirectional interconnections and a stepwise computation of the incoming information are thought to be the basics of memory storage and retrieval [21,22].

In the present study, we investigated the distribution of the phosphorylated tau protein (AT8) in 7 different tauopathies in 24 anatomically defined subregions or layers of the hippocampal formation and developed a mathematical algorithm to compare the patterns of the tau deposition. Here we demonstrate regional selective vulnerability patterns as distinguishing features of these disorders in functionally relevant structures of the hippocampus.

\section{Materials and Methods}

Selection of Cases

A total of 94 patients (39 men) were included in this study (online suppl. table 1; for all online suppl. material, see www.karger.com/doi/10.1159/000365548). These were recruited either from the tissue bank obtained from routine diagnostic practice of the Institute of Neurology (PSP, CBD, and PiD cases), or included cases (GGT, tau-astrogliopathy of the elderly, and AD-related pathology with or without AGD) from previously published studies [5, 6, 23]. The following tauopathies have been studied: (1) pure AD-related pathology (40 cases), divided into Braak and Braak (BB) stages I-VI of the neurofibrillary pathology [24, 25]; (2) AGD [26] associated with AD-related pathology, BBI-IV (18 cases: 2 with BBI, 9 with BBII, 3 with BBIII and 4 with BBIV); (3) PiD (8 cases); (4) CBD (6 cases); (5) PSP (7 cases); (6) uniform cases of GGT (5 cases of type 1 according to recent consensus nomenclature [4]) previously published as white matter tauopathy with globular glial inclusions [23], and (7) tau-astrogliopathy of the elderly [5] [10 cases; further stratified as 5 cases of the medial temporal lobe-predominant (MTL-predominant) group and 5 cases of the limbic-subcortical type; see below]. All cases have been evaluated in diagnostic sessions involving 2 or more certified neuropathologists following diagnostic criteria [27]. Cases with concomitant vascular pathology and prominent $\alpha$-synucleinopathy (other than minor concomitant $\alpha$-synuclein restricted to the amygdala, and one case with BBI of the Lewy body pathology) have been excluded. For non-AD, we included only those cases where immunostaining for amyloid- $\beta$ and Bielschowsky silver staining did not show a sufficient amount of plaques to diagnose AD at any level according to the National Institute on Aging-Alzheimer's Association criteria [28]. 
The overall distribution of tau pathology in the studied PSP or CBD cases was similar, thus we did not include early phases of PSP [29] or rare variants of CBD [27]. Samples were collected following local regulations and the study was approved by the Ethics Committee of the Medical University of Vienna (No. 396/2011).

\section{Topographical and Semiquantitative Analysis}

Formalin-fixed and paraffin-embedded tissue sections ( $3 \mu \mathrm{m}$ tick) were examined for tau pathology in the posterior hippocampus at the level of corpus geniculatum laterale using the AT8 antibody $11: 200$; Pearce Biotechnology, Rockford, Ill., USA). The AT8 antibody labels the most frequently phosphorylated tau epitope in tauopathies (Ser202, and to a minor extent Thr205) [30]. Although many sites of phosphorylable tau epitopes in tauopathies have been identified so far [1], it seems to be the most consistent one and hence standardized and used in neuropathologic routine [24, 25, 27].

We evaluated AT8 immunoreactivity (IR) in neurons (irrespectively of the morphology of the accumulation thus including diffuse cytoplasmic IR and neurofibrillary tangles), astrocytes, oligodendrocytes, and in the neuropil (comprising fine neurites and threads and fine granular staining) in 7 major regions of the hippocampus (the DG, CA1-4, subiculum) and the EC further divided into 24 subregions or layers according to anatomical criteria [31]. The regions and layers investigated included: upper and lower blade of the DG (each subdivided into 3 layers: stratum moleculare, stratum granulosum and stratum polymorphe), CA1-3 (stratum oriens, stratum pyramidale, and stratum radiatum as well as stratum lucidum in the CA3), the CA4, the subiculum, the stratum lacunosum-moleculare (at the level of the CA1) and the lateral and medial EC (each subdivided into outer layers 1-3 and inner layers 4-6). A semiquantitative scoring was performed as follows: negative (0); a weak/mild score (1) was given when barely any IR was present in the neuropil or just a few cells were labelled (under 30\%), and a moderate score (2) was given for a labelling easily recognized (fine neuritic/granular IR) and for cellular labelling, if an estimated $30-60 \%$ of the cells were labelled. The staining was scored as strong/severe (3), if it could be recognized even without the microscope (for fine neuritic/granular IR) or for cells, if it is estimated that over $60 \%$ of cells are labelled (online suppl. fig. 1). This scoring was highly reproducible and showed $100 \%$ interrater agreement. It was tested in a pilot fashion: both evaluators (I.M. and G.G.K.) examined blindly 30 randomly chosen cases. The anatomical regions were defined in sessions using a multi-headed microscope. Consequently, the statistical and mathematical analyses used the scores of one evaluator (I.M.). Semiquantitative scoring enabled to differentiate between distinct cell populations (neurons, astroglia and oligodendroglia), which would not be possible with other methods. In addition, semiquantitative scoring has been shown to correlate well with computer-assisted quantitative measurement [32].

\section{Statistical Analysis}

The statistical analysis was performed using SPSS version 20 (SPSS, Inc.). AT8-IR scores for neuronal and fine neuritic/granular IR, in cases with AD-related pathology, with or without AGD, were compared using the Mann-Whitney U test. A significance level of 0.05 was used.

\section{Mathematical Analysis}

The data of 94 patients (described in the section Selection of Cases) were used for the mathematical analysis with the program ' $R$ '. Each case could be presented as a 96-dimensional vector, determined by 4 evaluated variables (neuronal, astrocytic, oligodendroglial, and fine neuritic/granular AT8-IR) in 24 subregions or layers of the hippocampus ( $4 \times 24=96$ dimensions). As the diagnosis for each case was known, the reference vector for every disease (diagnosis) could be computed, representing the median values for a given diagnosis. In order to compare the constellation of AT8-IR (the combination of intensities of AT8-IR for each of the variables in all investigated subregions or layers), we applied an algorithm to provide a measure of relative 'distance' between reference vectors for each tauopathy. The distance represents how distinct the pattern of the AT8-IR is between different tauopathies, comparing both the spatial (different hippocampal subregions/layers) and the cellular (different cell types in these regions with or without neuropil) AT8-IR. Thus, similar AT8-IR constellations (similar disease entities) would result in shorter relative distance, whereas different constellations provide far distance. For technical details, see online supplementary methods. 
Dementia

and Geriatric

Cognitive Disorders

\begin{tabular}{l|l}
\hline \multicolumn{2}{l|}{ Dement Geriatr Cogn Disord 2014;87:375-388 } \\
\hline DOI: 10.1159/000365548 & $\begin{array}{l}\text { @ 2014 S. Karger AG, Basel } \\
\text { www.karger.com/dem }\end{array}$ \\
\hline
\end{tabular}

Milenkovic et al.: Patterns of Hippocampal Tau Pathology Differentiate

Neurodegenerative Dementias

\section{Results}

\section{Patterns of AT8-IR in the Hippocampus}

Cortical-Hippocampal-Predominant Tauopathies: PiD and AD-Related Pathology

PiD and AD-related pathology show different patterns of AT8-IR in the hippocampus. The CA1, CA2, subiculum and EC reveal already at early BB stages both neuronal and fine neuritic/ granular AT8-IR. This tau burden is increasing with higher BB stages (fig. 1a, b, fig. 2a-g). At late stages (BBV-VI), moderate involvement of the CA4 and to some extent CA3 can be observed (fig. 1a, b, fig. 2d-g). By contrast, in PiD, in addition to the CA1, CA2, subiculum and EC, the DG and the CA4 regions are strongly affected (fig. 1a, b, fig. $2 \mathrm{~h}-\mathrm{j}$ ). However, in PiD, the CA2 region shows severe neuronal but only moderate fine neuritic/granular AT8-IR in the strata pyramidale and radiatum (fig. 1b). By contrast, in the DG, severe neuronal AT8-IR (granule cells) can be seen (fig. 1a, fig. 2j). Further, abundant fine neuritic/granular AT8-IR is present in the polymorph layer (arrow in fig. 2j). Similarly, the CA4 region reveals a unique pattern of severe fine neuritic/granular AT8-IR with a lack of neuronal AT8 (fig. 1a, b, fig. 2i). In both AD-related neurofibrillary pathology and PiD, only minor glial AT8-IR is observed.

\section{Concomitant AGD Modifies the Pattern of AD-Related Tau Pathology}

In general, the distribution of AT8-IR is similar to the corresponding BB stages of the AD-related pathology (BBI-IV) without concomitant AGD. However, the pattern of the distribution reveals a mixture of different BB stages, ranging from stages III-IV to late stages (fig. 1a-d). Indeed, statistical analysis demonstrates higher tau burden in selected subregions/layers of the hippocampus in cases with concomitant AGD, as compared to pure AD-related pathology (table 1). The following regions show an 'upgrade' in the tau load (either or both neuronal or fine neuritic/granular pattern) in cases with a concomitant presence of AGD: DG, CA1, subiculum, and EC. In addition, occasional astrocytic AT8-IR in the stratum lacunosum-moleculare of the CA1/subiculum can be observed. Furthermore, moderate oligodendroglial AT8-IR is noted in the EC white matter (fig. 1d).

\section{Subcortical-Predominant Tauopathies: PSP and CBD}

Both CBD and PSP cases show a uniform pattern characterized by moderate AT8-IR in the hippocampus. CBD and PSP reveal a similar distribution of neuronal AT8 (stratum pyramidale of the CA1 and the CA2, as well as in the CA4, the subiculum and the lateral EC). However, CBD shows moderate neuronal AT8 deposition in the stratum pyramidale of the CA3, contrasting PSP cases. In addition, more prominent AT8-IR in the granule cells can be noted.

Fig. 1. The AT8 signature of tauopathies in the hippocampus. Note distinct AT8-IR patterns for different tauopathies. AT8-IR load is color-coded: light blue represents no IR, red represents strong IR (e). In AD cases, note early highlighting of the hot spots (CA1, CA2, subiculum and EC) with increasing AT8-IR load for neuronal (a) and fine neuritic/granular (b) deposits. By contrast, only occasional astroglial (c) and oligodendroglial (d) IR is observed. PiD shows a different AT8-IR pattern with prominent involvement of the DG, CA4, CA1 and subiculum (hot spots), as highlighted by a red color code. Note the change in the AT8-IR pattern of the AD-related pathology with concomitant AGD (a, b). CBD and PSP cases showed more prominent neuronal AT8-IR in the stratum oriens and radiatum of CA1, CA2 and CA3, as compared to early BB stages (a). Note the severe affection of the astrocytes in MTL-predominant tau-astrogliopathy of the elderly (elderly tau-MTL) in the DG, CA4 but also moderate affection in the EC, contrasting all other tauopathies (c). Observation of mild to moderate oligodendroglial AT8-IR in different regions (strata oriens of the CA1, CA2, CA3, but also in the stratum lacunosum-moleculare and in the EC) should raise a suspicion for CBD (d). By contrast, GGT is characterized by a strong presence of oligodendroglial AT8-IR in many areas, especially in the white matter of the EC (d). The asterisk marks regions with mean values of 0.1 . Colors refer to the online vesion only. 
Dementia

and Geriatric
Cognitive Disorders
Dement Geriatr Cogn Disord 2014;87:375-388
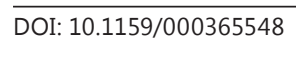

Milenkovic et al.: Patterns of Hippocampal Tau Pathology Differentiate Neurodegenerative Dementias

www.karger.com/dem

a

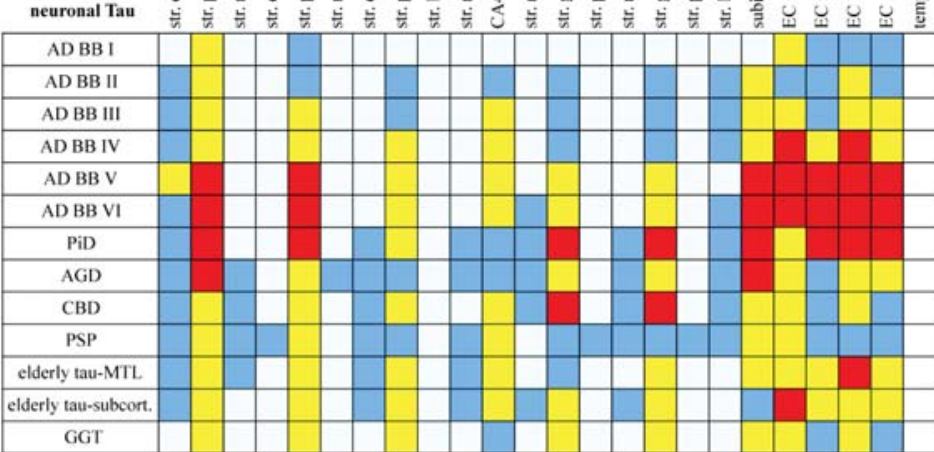

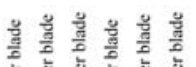

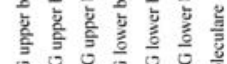

C 2014 S. Karger AG, Basel

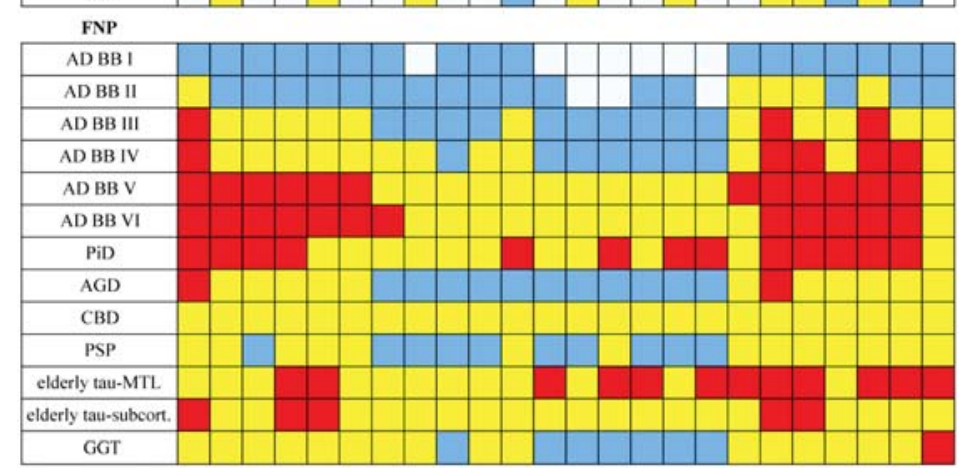

b

उ.

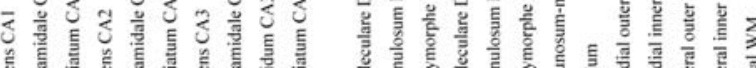

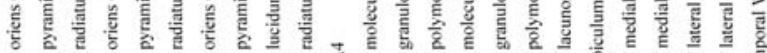

neuronal Tau
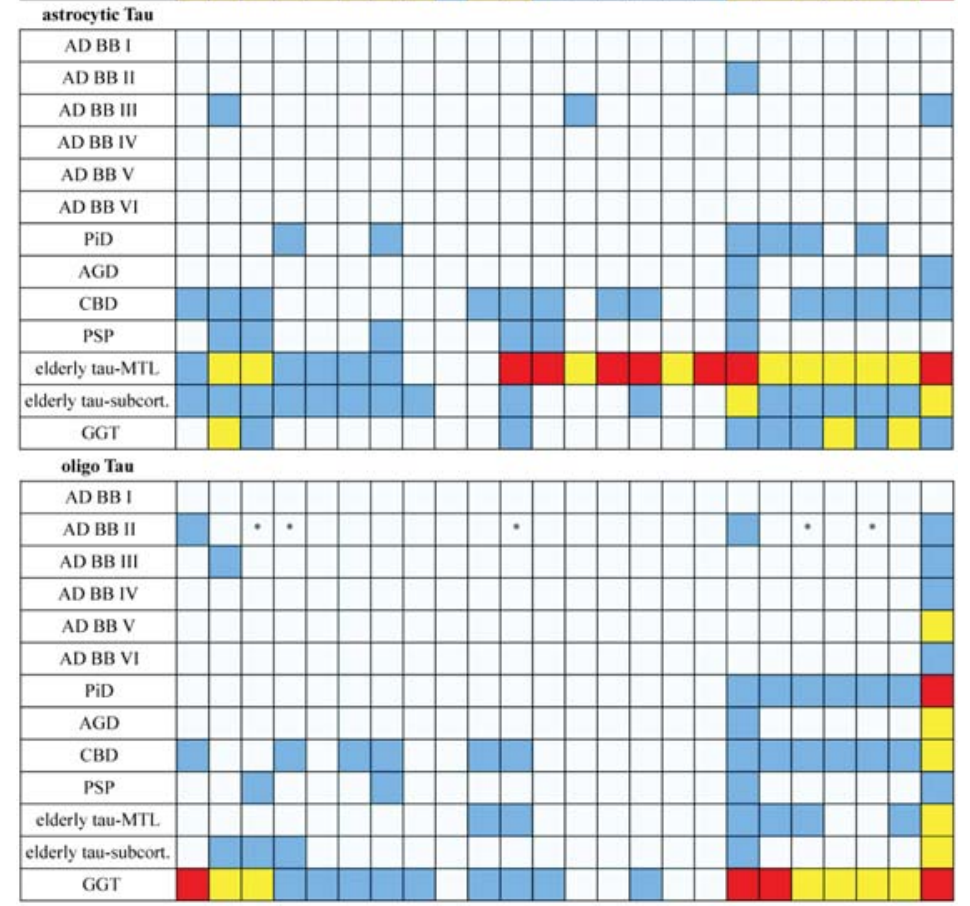

e

severe

moderate

1.99-1.0 $0.99-0.1$

no IR
0 

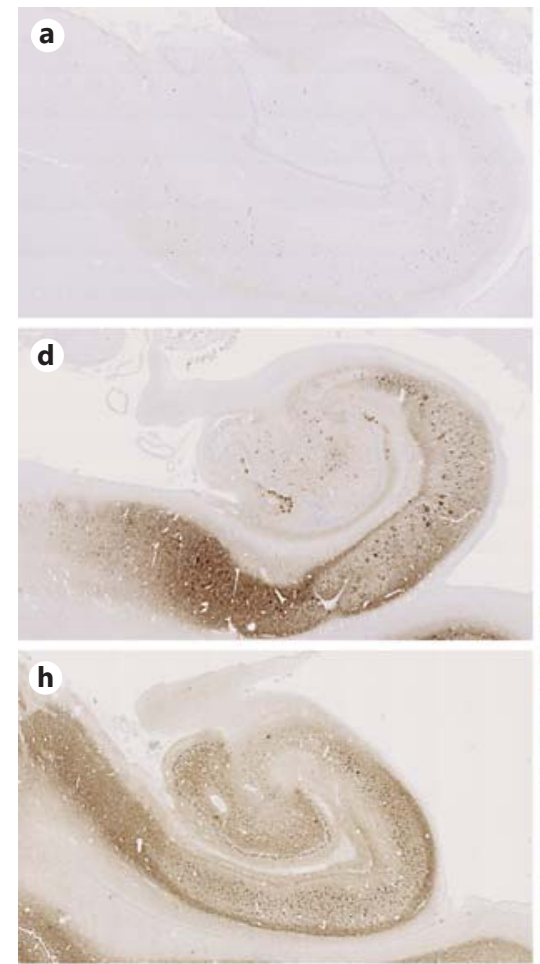

k
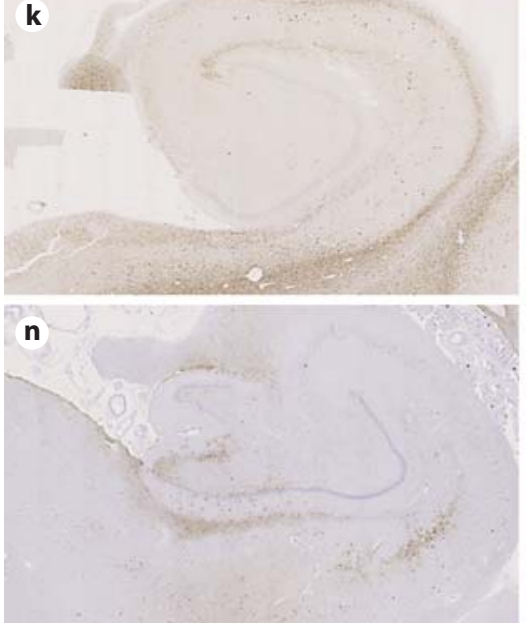
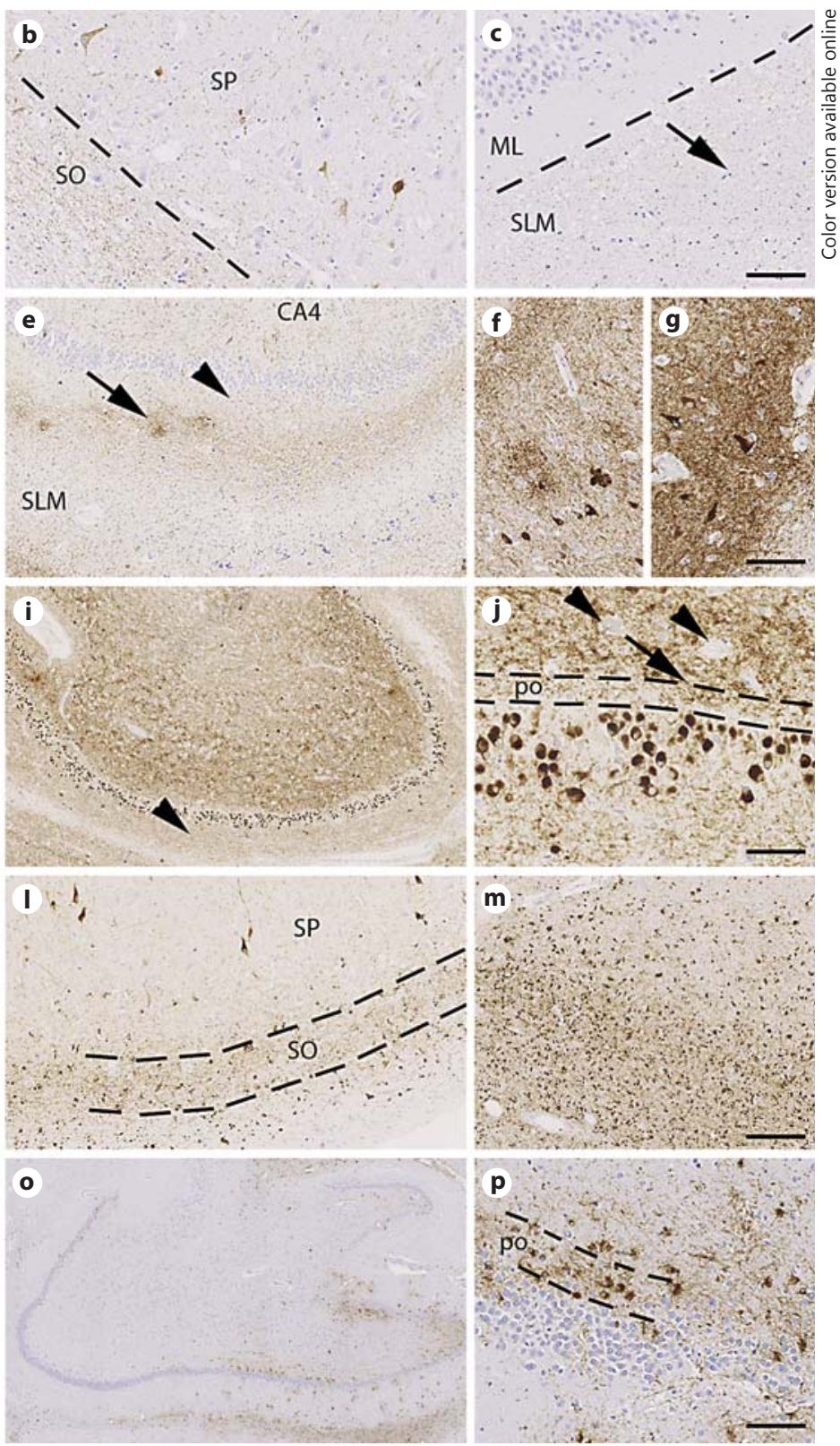

Fig. 2. Distribution of the AT8-IR in the hippocampus. a-c In stage BBII of AD-related pathology, mild affection of the hippocampus, predominantly in the CA1 (b) and the subiculum, can be observed. Stratum lacunosum-moleculare (SLM) shows already here AT8-IR dots in the perforant path targeting zone (c, black arrow), without significant affection of the DG. $\mathbf{d}-\mathbf{g}$ Stage BBVI of AD-related pathology is characterized by prominent AT8-IR throughout the hippocampus. Although the perforant path targeting zone is strongly affected (e, black arrow), the inner $1 / 3$ of the stratum moleculare (e, black arrowhead) and granule cells of the DG showed only occasional IR (e). By contrast, both the stratum pyramidale (f) and stratum oriens (g) of the CA1 are severely affected. $\mathbf{h}-\mathbf{j}$ Prominent involvement of the hippocampus in PiD. Notably, the DG is strongly affected (i), including the whole stratum moleculare (black arrowhead) and the granule cells. In the CA4, a strong fine neuritic/granular pattern is observed (black arrow), with practically lacking neuronal IR (j; black arrowheads indicate not stained neurons in the CA4; po = stratum polymorphe). $\mathbf{k}-\mathbf{m}$ GGT shows prominent involvement of oligodendroglia in the hippocampus. These can be observed in many regions, including the stratum oriens (SO) of the CA1 (I) and the white matter of the EC ( $\mathbf{m})$. SP = Stratum pyramidale. $\mathbf{n}-\mathbf{p}$ The medial temporal lobe-predominant subtype of the tau-astrogliopathy of the elderly shows strong presence of astrocytes in the hippocampus, particularly in the stratum polymorphe (po) of the DG (o, p) and CA4 (p). Scale bar: $1 \mathrm{~mm}(\mathbf{a}, \mathbf{d}, \mathbf{h}, \mathbf{k}, \mathbf{n}) ; 500 \mu \mathrm{m}(\mathbf{b}, \mathbf{e}, \mathbf{i}, \mathbf{I}, \mathbf{m}, \mathbf{o}) ; 100 \mu \mathrm{m}(\mathbf{f}, \mathbf{g}, \mathbf{j}, \mathbf{p})$. 
Dementia

Cognitive Disorders

Table 1. Influence of concomitant AGD on tau burden in the hippocampus compared to pure AD-related pathology of different BB stages

\begin{tabular}{l|l}
\hline \multicolumn{2}{|l}{ Dement Geriatr Cogn Disord 2014;87:375-388 } \\
\hline DOI: $10.1159 / 000365548$ & $\begin{array}{l}\text { C 2014 S. Karger AG, Basel } \\
\text { www.karger.com/dem }\end{array}$ \\
\hline
\end{tabular}

Milenkovic et al.: Patterns of Hippocampal Tau Pathology Differentiate Neurodegenerative Dementias

\begin{tabular}{|c|c|c|c|c|c|}
\hline & & BBI & BBII & BBIII & BBIV \\
\hline $\begin{array}{l}\text { Upper } \\
\text { blade DG }\end{array}$ & $\begin{array}{l}\text { str. moleculare } \\
\text { str. granulosum } \\
\text { str. polymorphe }\end{array}$ & $\begin{array}{l}-/- \\
-/- \\
-/-\end{array}$ & $\begin{array}{l}-/- \\
* /- \\
-/ *\end{array}$ & $\begin{array}{l}-/- \\
* /- \\
-/-\end{array}$ & $\begin{array}{l}-/- \\
-/- \\
-/-\end{array}$ \\
\hline $\begin{array}{l}\text { Lower } \\
\text { blade DG }\end{array}$ & $\begin{array}{l}\text { str. moleculare } \\
\text { str. granulosum } \\
\text { str. polymorphe }\end{array}$ & $\begin{array}{l}-/- \\
-/- \\
-/-\end{array}$ & $\begin{array}{l}-/- \\
* / * \\
-/ *\end{array}$ & $\begin{array}{l}-/- \\
* /- \\
-/-\end{array}$ & $\begin{array}{l}-/- \\
-/- \\
-/-\end{array}$ \\
\hline CA1 & $\begin{array}{l}\text { str. oriens } \\
\text { str. pyramidale } \\
\text { str. radiatum }\end{array}$ & $\begin{array}{l}-/- \\
-/- \\
-/-\end{array}$ & $\begin{array}{l}-/- \\
* / * \\
-/^{*}\end{array}$ & $\begin{array}{l}-/- \\
-/^{*} \\
-/-\end{array}$ & $\begin{array}{l}-/- \\
-/- \\
-/-\end{array}$ \\
\hline CA2 & $\begin{array}{l}\text { str. oriens } \\
\text { str. pyramidale } \\
\text { str. radiatum }\end{array}$ & $\begin{array}{l}-/- \\
-/- \\
-/-\end{array}$ & $\begin{array}{l}-/^{*} \\
* / * \\
-/^{*}\end{array}$ & $\begin{array}{l}-/- \\
-/- \\
-1-\end{array}$ & $\begin{array}{l}-/- \\
-/- \\
-/-\end{array}$ \\
\hline CA3 & $\begin{array}{l}\text { str. oriens } \\
\text { str. pyramidale } \\
\text { str. lucidum } \\
\text { str. radiatum }\end{array}$ & $\begin{array}{l}-/- \\
-/- \\
-/- \\
-/-\end{array}$ & $\begin{array}{l}-/- \\
-/^{*} \\
-/- \\
-/^{*}\end{array}$ & $\begin{array}{l}-/^{*} \\
-/- \\
-/^{*} \\
-/-\end{array}$ & $\begin{array}{l}-/- \\
* /- \\
-/- \\
-/-\end{array}$ \\
\hline $\begin{array}{l}\text { str. lacunc } \\
\text { CA4 } \\
\text { subiculun } \\
\text { EC medial } \\
\text { EC medial } \\
\text { EC lateral } \\
\text { EC lateral } \\
\text { White ma }\end{array}$ & $\begin{array}{l}\text { sum-moleculare } \\
\text { outer layers } \\
\text { inner layers } \\
\text { outer layers } \\
\text { inner layers } \\
\text { tter EC }\end{array}$ & $\begin{array}{l}-/- \\
-/- \\
* /- \\
-/- \\
-/- \\
-/- \\
-/- \\
-/-\end{array}$ & $\begin{array}{l}-/- \\
-/- \\
* / * \\
* /- \\
-/ * \\
-/- \\
-/- \\
-/-\end{array}$ & $\begin{array}{l}* /- \\
-/ * \\
-/- \\
-/- \\
-/- \\
-/- \\
-/- \\
-/ *\end{array}$ & $\begin{array}{l}-/ * \\
-/- \\
-/- \\
-/^{*} \\
-/- \\
-/^{*} \\
-/- \\
-/-\end{array}$ \\
\hline
\end{tabular}

The first symbol represents neuronal tau burden, the second one fine neuritic/fine granular immunostaining pattern. Regions not involved directly in AD-related pathology (DG, CA2) are affected if there was a concomitant AGD pathology. - = No difference between groups; $*$ = significant difference between groups $(\mathrm{p}<0.05)$.

A further distinguishing feature of CBD is affection of all layers in the CA3 region in the form of moderate fine neuritic/granular AT8-IR as well as in the DG (fig. 1b). By contrast, PSP cases reveal less fine neuritic/granular AT8-IR in the DG (fig. 1a). Although both CBD and PSP show weak to moderate glial AT8 deposits, these are more prominent in CBD. These include more prominent astrocytic AT8-IR in the stratum oriens of the CA1 as well as in the EC (fig. 1c). Further, CBD cases reveal a weak but constant presence of oligodendroglial AT8-IR in the strata orientia of the CA1-3 and in the EC (fig. 1d).

Further Tauopathies: Tau-Astrogliopathy in the Elderly and GGT

Based on the distribution of non-AD type AT8-IR in elderly brains, we identified four major patterns in a recent study [6]. Focusing on the hippocampal pattern of AT8-IR, we included two major groups in this study: one which predominates in the MTL (MTL-predominant group; previously described as hippocampal-DG-amygdala-predominant/granular and thorny astrogliopathy and neuronal tauopathy type) and one which also involves the subcortical regions termed here as limbic-subcortical type (limbic-basal ganglia-nigral/granular and thorny astrogliopathy and neuronal tauopathy type) [6]. 


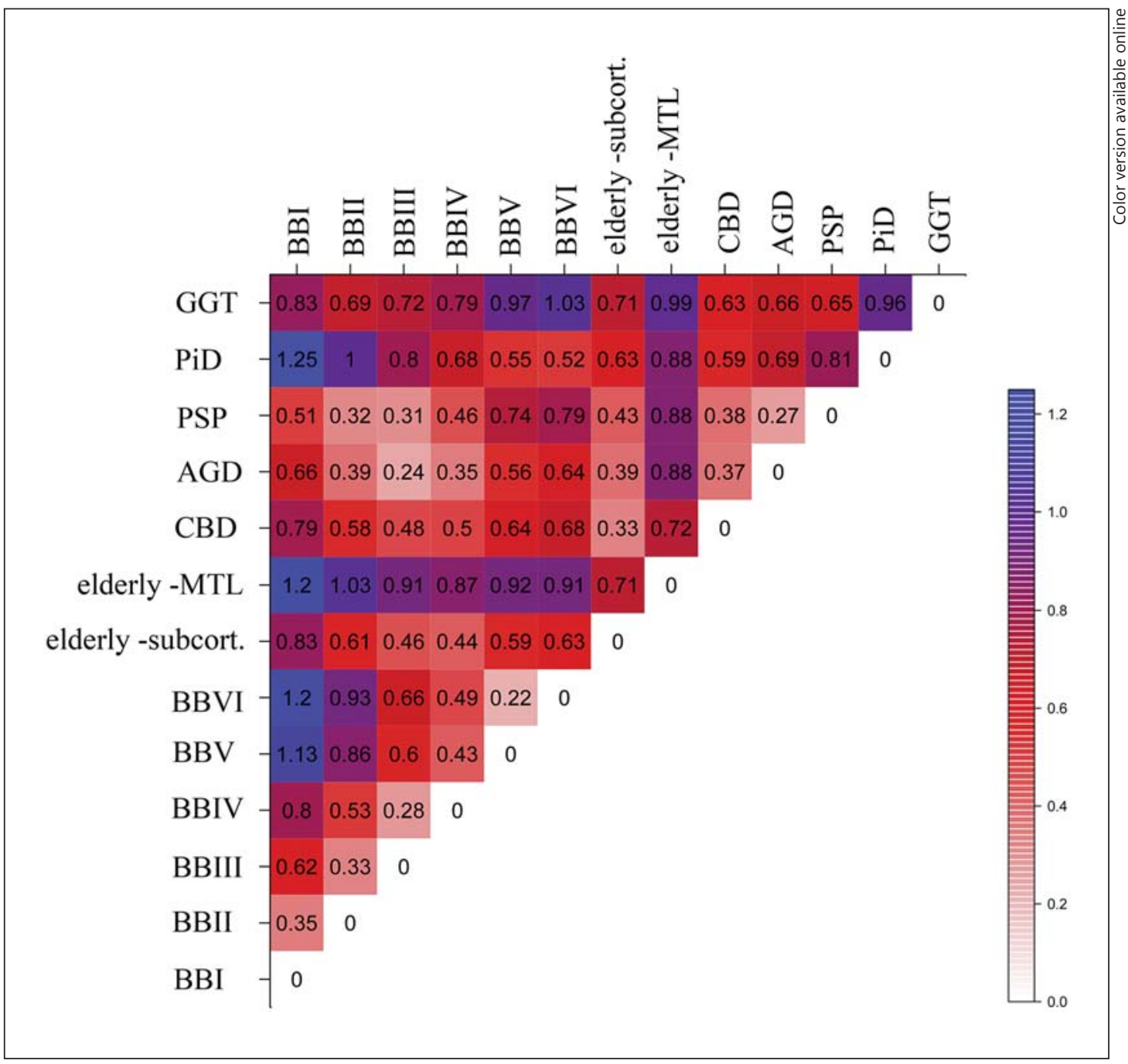

Fig. 3. Color-coded schematic representation of the relative distances between tauopathies. Based on the mathematical model, relative distances are shown in color code, ranging from white (very close) to dark blue (very distant). The distance of each disease to itself is 0 . Note a stepwise increase in distance with increasing BB stages (BBI-VI). The distance is very close between all neighboring stages, suggesting only subtle additional tau pathology in consecutive BB stages. The limbic-subcortical subtype of tau-astrogliopathy of the elderly (elderly - subcortical), CBD, PSP, and AGD show close distance to each other. The most distant diseases to all others is the MTL-predominant tau-astrogliopathy of the elderly (elderly-MTL), followed by GGT, and PiD. Noteworthy, concomitant AGD changes the distance to other diseases, as compared to pure AD-related pathology of early BB stages. Distances are represented as a 10-fold value for easier comparison.

The distinguishing feature of the MTL-predominant subtype from all other tauopathies (including the limbic-subcortical subtype) is the unique involvement of the DG and the CA4. All layers of the DG, particularly the stratum polymorphe, show a high density of astrocytic AT8-IR with characteristic morphology, i.e. small clusters of thorn-shaped astrocytes with perinuclear accentuation of AT8-IR (fig. 1c, fig. $2 \mathrm{n}-\mathrm{p}$ ). This is followed by moderate astrocytic AT8-IR in the CA1 and in the EC. CA2 and CA3 reveal only weak astrocytic AT8 abundance (fig. 1c, fig. 2n). Further, fine neuritic/granular AT8-IR in the form of patchy deposits can be found in the vicinity of the AT8-IR astrocytes. 
The limbic-subcortical type of tau-astrogliopathy of the elderly shows a similar, but less pronounced pattern of the astrocytic AT8-IR in the examined regions (fig. 1c). For instance, here almost no astrocytic AT8 deposits can be observed in the DG. The only exception is the stratum moleculare, where only occasional AT8-IR astrocytes can be observed (fig. 1c). By contrast, more pronounced neuronal AT8-IR is noted in the DG, as compared to the MTLpredominant subtype. This includes more pronounced AT8-IR in the granule cells as well as more AT8-positive neurons in the stratum moleculare (fig. 1b). Both the MTL-predominant subtype and the limbic-subcortical type of tau-astrogliopathy of the elderly reveal only occasional oligodendroglial AT8-IR (fig. 1d).

GGT is characterized by prominent oligodendroglial AT8-IR predominantly in white matter regions (fig. $2 \mathrm{k}-\mathrm{m}$ ). The white matter of the EC (fig. $1 \mathrm{~b}, \mathrm{~d}$, fig. $2 \mathrm{~m}$ ) is severely affected [23]. Moderate neuronal tau pathology can be found in the stratum pyramidale of the CA1. In other regions, only minor neuronal AT8-IR is present (fig. 1a). Several regions show moderate fine neuritic/granular AT8-IR (CA1-4, subiculum, EC). Notably, the DG is characterized only by weak fine neuritic/granular AT8-IR (fig. 1b, fig. 2k). Gray matter astrocytes exhibit a starlike appearance with globular dot-like AT8-IR in proximal segments of the processes.

\section{Hippocampal AT8-IR Patterns Are Disease-Characteristic, as Revealed by Mathematical}

Cluster Analysis of AT8-IR Patterns

Our semiquantitative data suggested disease-specific patterns of the tau deposition in hippocampal regions. Therefore, we created a mathematical comparison model to investigate the relative distance between diseases.

Figure 3 summarizes the data obtained. Similar tau constellations and yet a stepwise increase in the distance between different $\mathrm{BB}$ stages of AD-related tau pathology demonstrate the power of our algorithm. All BB stages reveal a far distance to all other tauopathies (fig. 3).

As already suggested in figure 1, the signature of PiD in the hippocampus is different from all other tauopathies investigated. Although far from all other diseases, the closest distance can be measured to BBV (distance 0.55) and BBVI (distance 0.52).

Our algorithm reveals a relatively moderate distance between the limbic-subcortical type of the tau-astrogliopathy of the elderly and CBD, followed by AGD and PSP. The distance to BBII and BBIII is larger (fig. 3). The MTL-predominant subtype of the tau-astrogliopathy shows a far distance to all other tauopathies (fig. 3).

Cases with concomitant AGD show the closest distance to BBIII, although the majority of these cases were originally classified as presenting with lower BB stages. Interestingly, AGD, PSP, CBD and the limbic-subcortical type of the tau-astrogliopathy of the elderly are very close in distance, comparable to neighboring BB stages (fig. 3). GGT is characterized by a far distance to all other tauopathies. The closest distance can be measured to CBD, AGD, and PSP (fig. 3).

\section{Discussion}

A growing body of evidence suggests that tauopathies may be characterized by selective cellular and regional vulnerability, which might eventually influence the clinical presentations [32-36]. Our semiquantitative approach allowed us to distinguish different cell-related patterns of AT8-IR, which can be missed with a morphometric method evaluating only the total load of tau pathology. Here we present, to our knowledge, the first detailed comparative study on AT8-IR in the hippocampus in 7 major tauopathies. Our data strongly suggest: (1) disease-specific AT8-IR in the hippocampus, which may (2) be influenced and shifted towards other regions by co-occurrence of tauopathies (i.e. AGD and AD-related tau pathology). These 
regions may be designated as hot spots for the disease and might influence the clinical presentations due to cellular dysfunction associated with pathological tau deposition. In addition, our data revealed that (3) AT8-IR patterns in the hippocampus are disease characteristic, even in tauopathies, which primarily do not affect the hippocampus. Thus, knowledge of hippocampal vulnerability patterns may elucidate the understanding of some cognitive aspects of tauopathies and may serve as a rationale for the development of better diagnostic test batteries and eventually targeted therapy aiming to improve certain hippocampusrelated memory functions.

AT8 has an advantage towards other phosphorylation-dependent antibodies or silver stains, as it labels both pretangles (nonargyrophilic and nonubiquitinated) and tangles in all cell populations, including tau pathology in glia [25]. By contrast, other disease-related phosphorylation sites appear either in a specific temporally dependent manner [30,37], exhibit variable phosphorylation [38] or may even be disease-specific [39] and are therefore not eligible for comparison between different diseases.

Studies on animal models assigned special functions to different anatomical subregions in the hippocampus [40]. For instance, the DG is implicated in spatial pattern separation [40], whereas the CA3 is responsible for pattern association, spatial and temporal pattern completion and spatial and temporal working memory [41]. The CA1 region is shown to be involved in temporal pattern separation [41]. Although a direct correlation between morphology and function cannot be established in humans, different test batteries revealed hippocampal involvement in humans in all functions found in animal models as well [42].

Our data revealed that cortical-predominant tauopathies (and dementias) share some hot spots in the hippocampus, whereas some distinctive hot spots can be defined as well. The constellation of the AT8-IR in the hippocampus is highly disease-specific, as measured by a large distance between PiD and all BB stages (fig. 3). Indeed, in AD-related tau pathology a continuous increase in the distance between neighboring BB stages can be detected, supporting the stepwise progression of tau pathology. Interestingly, both $\mathrm{AD}$ and PiD (i.e. cortical type dementias) share as a hot spot the subiculum, as one of the most important afferent systems towards association cortices [43], the CA1 and the EC. Thus, the affection of the subiculum may dissociate the hippocampus from the rest of the brain, whereas the early affection of outer layers of the EC (including layer II) may disturb the cortical input to the hippocampus. Further, both AD and PiD may be characterized by an impaired temporal pattern separation. The most striking difference, however, seems to be the affection of the DG granule cells as well as the CA4 (fine granular/neuritic AT8-IR with lacking neuronal IR) in PiD when compared to AD-related pathology. Since DG is involved in spatial pattern separation [40], this might suggest its distinct impairment in PiD. However, whether vulnerability of the DG granule cells depends on the molecular feature of tau protein (3R predominance in PiD vs. mixed 3R/4R tau in AD-related pathology) merits further investigation.

Importantly, concomitant AGD (4R tauopathy) shifts the hot spot of the AD-related tau pathology towards the DG. As already suggested by other groups [26], we noted that concomitant AGD increases neuronal AT8-IR scores in the hippocampus. Noteworthy, our data revealed that this is not only the case in the CA1, but also in the DG, CA2, subiculum and in the inner layers of the medial EC (table 1). This would not only suggest a pathophysiological interaction between neuropil and neuronal deposits, but may further support the notion of lowering the threshold for cognitive decline in concomitant AGD $[6,44]$.

We have recently reported a group of tauopathies in the elderly, which cannot be clearly diagnosed by current diagnostic criteria $[5,6]$. Focusing only on the hippocampal AT8-IR patterns, the MTL-predominant group and limbic-subcortical-predominant group of the tauastrogliopathy of the elderly were included in the present study [6]. Variable hippocampal pathology in neurodegenerative diseases with involvement of subcortical structures (i.e. PSP, 
Milenkovic et al.: Patterns of Hippocampal Tau Pathology Differentiate

Neurodegenerative Dementias

CBD, and limbic-subcortical type of tau-astrogliopathy of the elderly) may indicate that they represent a spectrum [45-48]. Indeed, the short distances between CBD, PSP, AGD and even the limbic-subcortical type of tau-astrogliopathy of the elderly would support this concept. On the other hand, this would suggest that AGD, CBD, and PSP have some overlapping features as suggested previously [49]. However, our data suggest that they can also be clearly distinguishable based on their hippocampal AT8-IR patterns (see also the heat map, fig. 1). Interestingly, CBD, PSP, and AGD showed affection of the DG granule cells (which was not observed in AD-related pathology), which was less prominent as compared to PiD. Thus, DG granule cells seem to show early vulnerability for tauopathies with single isoform predominance (i.e. 3R tauopathy PiD or 4R tauopathies PSP, CBD, and AGD), irrespective of whether the brunt of tau pathology is subcortical or cortical; this early harmfulness is not seen in AD-related pathology (i.e. mixed isoforms $3 R+4 R$ ).

Although PSP and CBD do not affect the hippocampus primarily, distinctive patterns of AT8-IR can be observed: CBD, for instance, showed a higher rate of oligodendroglial AT8 deposits in the stratum oriens of the CA1, CA2 and CA3, but also in the CA4, as compared to similar BB stages or other tauopathies. This was confirmed by our mathematical analysis, which showed a far distance between CBD and different BB stages. These data would further suggest a CBD-characteristic AT8 signature in the hippocampus. Similarly to CBD cases, PSP was characterized by neuronal AT8-IR in the stratum oriens and radiatum throughout the CA regions, but also in the stratum polymorphe of the DG compared to other diseases. These regions contain mainly putative inhibitory GABAergic interneurons [50,51], which play an important role in regulating and synchronizing hippocampal neuronal oscillations [52]. AT8-IR patterns in PSP were relatively close to BBII, BBIII, CBD, and AGD. Noteworthy, the distance to BBII and BBIII was similar (0.32 to BBII and 0.31 to BBIII) and comparable to the distance between BBII and BBIII (0.35), suggesting a characteristic hippocampal AT8-IR pattern in PSP, which can be distinguished from early BB stages. Although the focus of our study is not to provide diagnostic guidelines based on the evaluation of the hippocampus, our observations strongly suggest that when evaluating only the hippocampus for screening during the diagnostic procedure, the above-mentioned distinguishing features should alert for further systematic mapping of tau pathology in other anatomical areas.

The MTL-predominant group of the tau-astrogliopathy of the elderly revealed a large distance towards all other tauopathies investigated. The DG and the CA4 were highlighted as hot spots for the MTL-predominant group of the tau-astrogliopathy of the elderly. However, in contrast to all other tauopathies, the astrocytes were severely affected, whereas only moderate neuronal AT8-IR was observed. Nevertheless, severe affection of the astrocytes may impair the function of the neurons. Although the role of astrocytes in synaptic transmission has been a matter of debate [53-57], they may modulate synaptic transmission via tripartite synapses [58] and long-term potentiation $[59,60]$ in the hippocampus, which is thought to be a substrate for learning. In summary, for both types of tauopathy of the elderly (limbic-subcortical type and MTL type) included in this study, our observations support the concept that they are distinct from other forms of tauopathies. This is corroborated by their distinct features like subregional involvement and cellular preference of tau pathology (astrogliopathy) in the hippocampus. Moreover, tau pathology itself including phospho-specific sites of tau phosphorylation, tau conformation, tau truncation, and ubiquitination and modifications of the astroglial cytoskeleton shows distinct features as well [36].

The present study provides further evidence that GGTs (i.e. of type 1) are distinct tauopathy entities showing a unique distribution of tau pathology also in the hippocampus. Recently, GGTs have been grouped into three forms [4]; from these we included those where the uniform feature of widespread oligodendroglial tau pathology predominates [23]. Our observations show that the unique involvement of hippocampal white matter may isolate the 
Dementia

and Geriatric

Cognitive Disorders \begin{tabular}{l|l}
\hline \multicolumn{2}{l|}{ Dement Geriatr Cogn Disord 2014;87:375-388 } \\
\hline DOI: 10.1159/000365548 & $\begin{array}{l}\text { @ 2014 S. Karger AG, Basel } \\
\text { www.karger.com/dem }\end{array}$ \\
\hline
\end{tabular}

Milenkovic et al.: Patterns of Hippocampal Tau Pathology Differentiate

Neurodegenerative Dementias

hippocampus from the functionally relevant connections leading to severe cognitive decline. Further, the distribution of neuronal tau pathology is also clearly different in GGT, supporting a different origin from that seen in other tauopathies.

In addition to possible differences in clinical phenotypes, distinct cellular and regional tau constellations my influence the detection levels of this protein in cerebrospinal fluid [1]. One limitation of our study is that we could not correlate cerebrospinal fluid levels of tau/ phospho-tau with the regional distribution of the phosphorylated tau protein. An additional prospective study is needed to clarify this issue and also how subtle neuropsychological differences may reflect the selective vulnerability patterns shown here. It must be noted that we focused on the posterior part of the hippocampus, which has different projection patterns and different physiology in memory than the anterior part.

\section{Conclusions}

Comprehensive mapping of tau pathology in different tauopathies demonstrates different patterns of anatomical and cellular vulnerability. Our study (1) suggests disease-specific hot spots, arguing for regional selective vulnerability; (2) shows that AD-related tau pathology is strongly influenced by concomitant AGD, involving even subregions unaffected in pure AD-related pathology; (3) reveals that tau-astrogliopathies in the elderly and GGTs are distinct entities supported also by differences in the modifications of tau in glial cells [36], and (4) suggests that involvement of the hippocampus in PSP, CBD, PiD and GGT can be clearly distinguished from early AD-related changes.

These observations emphasize the need for better clinical stratification of patients with cognitive decline. Since the hippocampus has a very complex system of neurotransmitter inputs and neuronal groups expressing various receptors, the exact fingerprinting of pathology might serve as a rationale to develop more selective diagnostic test batteries.

\section{Acknowledgement}

This study was supported by the European Commission's 7th Framework Programme under GA No. 278486, 'DEVELAGE'.

\section{Disclosure Statement}

The authors report no conflict of interest.

\section{References}

1 Kovacs GG, Botond G, Budka H: Protein coding of neurodegenerative dementias: the neuropathological basis of biomarker diagnostics. Acta Neuropathol 2010;119:389-408.

2 Lee VM, Goedert M, Trojanowski JQ: Neurodegenerative tauopathies. Annu Rev Neurosci 2001;24:11211159.

3 Jellinger KA, Attems J: Neurofibrillary tangle-predominant dementia: comparison with classical Alzheimer disease. Acta Neuropathol 2007;113:107-117.

4 Ahmed Z, Bigio EH, Budka H, Dickson DW, Ferrer I, Ghetti B, Giaccone G, Hatanpaa KJ, Holton JL, Josephs KA, Powers J, Spina S, Takahashi H, White CL 3rd, Revesz T, Kovacs GG: Globular glial tauopathies (GGT): consensus recommendations. Acta Neuropathol 2013;126:537-544.

5 Kovacs GG, Molnar K, Laszlo L, Strobel T, Botond G, Honigschnabl S, Reiner-Concin A, Palkovits M, Fischer P, Budka H: A peculiar constellation of tau pathology defines a subset of dementia in the elderly. Acta Neuropathol 2011;122:205-222. 
Milenkovic et al.: Patterns of Hippocampal Tau Pathology Differentiate

Neurodegenerative Dementias

6 Kovacs GG, Milenkovic I, Wohrer A, Hoftberger R, Gelpi E, Haberler C, Honigschnabl S, Reiner-Concin A, Heinzl H, Jungwirth S, Krampla W, Fischer P, Budka H: Non-Alzheimer neurodegenerative pathologies and their combinations are more frequent than commonly believed in the elderly brain: a community-based autopsy series. Acta Neuropathol 2013;126:365-384.

7 Goedert M, Spillantini MG, Potier MC, Ulrich J, Crowther RA: Cloning and sequencing of the cDNA encoding an isoform of microtubule-associated protein tau containing four tandem repeats: differential expression of tau protein mRNAs in human brain. EMBO J 1989;8:393-399.

8 Buee L, Delacourte A: Comparative biochemistry of tau in progressive supranuclear palsy, corticobasal degeneration, FTDP-17 and Pick's disease. Brain Pathol 1999;9:681-693.

9 de Silva R, Lashley T, Strand C, Shiarli AM, Shi J, Tian J, Bailey KL, Davies P, Bigio EH, Arima K, Iseki E, Murayama S, Kretzschmar H, Neumann M, Lippa C, Halliday G, MacKenzie J, Ravid R, Dickson D, Wszolek Z, Iwatsubo T, Pickering-Brown SM, Holton J, Lees A, Revesz T, Mann DM: An immunohistochemical study of cases of sporadic and inherited frontotemporal lobar degeneration using 3R- and 4R-specific tau monoclonal antibodies. Acta Neuropathol 2006;111:329-340.

10 Song YJ, Halliday GM, Holton JL, Lashley T, O’Sullivan SS, McCann H, Lees AJ, Ozawa T, Williams DR, Lockhart PJ, Revesz TR: Degeneration in different parkinsonian syndromes relates to astrocyte type and astrocyte protein expression. J Neuropathol Exp Neurol 2009;68:1073-1083.

11 Santpere G, Ferrer I: Delineation of early changes in cases with progressive supranuclear palsy-like pathology. Astrocytes in striatum are primary targets of tau phosphorylation and GFAP oxidation. Brain Pathol 2009;19: 177-187.

12 Parent A: Carpenter's Human Anatomy. Baltimore, Williams \& Wilkins, 1996, pp 760-773.

13 O'Keefe J, Nadel L: The Hippocampus as a Cognitive Map. Oxford, Clarendon, 1978.

14 Lavenex PB, Amaral DG, Lavenex P: Hippocampal lesion prevents spatial relational learning in adult macaque monkeys. J Neurosci 2006;26:4546-4558.

15 Eichenbaum H, Dudchenko P, Wood E, Shapiro M, Tanila H: The hippocampus, memory, and place cells: is it spatial memory or a memory space? Neuron 1999;23:209-226.

16 Morris RG, Garrud P, Rawlins JN, O’Keefe J: Place navigation impaired in rats with hippocampal lesions. Nature 1982;297:681-683.

17 Nadel L: The hippocampus and space revisited. Hippocampus 1991;1:221-229.

18 O'Keefe J, Dostrovsky J: The hippocampus as a spatial map. Preliminary evidence from unit activity in the freely-moving rat. Brain Res 1971;34:171-175.

19 Scoville WB, Milner B: Loss of recent memory after bilateral hippocampal lesions. J Neurol Neurosurg Psychiatry 1957;20:11-21.

20 Squire LR: Memory and the hippocampus: a synthesis from findings with rats, monkeys, and humans. Psychol Rev 1992;99:195-231.

21 Colgin LL, Moser EI, Moser MB: Understanding memory through hippocampal remapping. Trends Neurosci 2008;31:469-477.

22 Moser EI: The multi-laned hippocampus. Nat Neurosci 2011;14:407-408.

23 Kovacs GG, Majtenyi K, Spina S, Murrell JR, Gelpi E, Hoftberger R, Fraser G, Crowther RA, Goedert M, Budka H, Ghetti B: White matter tauopathy with globular glial inclusions: a distinct sporadic frontotemporal lobar degeneration. J Neuropathol Exp Neurol 2008;67:963-975.

24 Alafuzoff I, Arzberger T, Al-Sarraj S, Bodi I, Bogdanovic N, Braak H, Bugiani O, Del-Tredici K, Ferrer I, Gelpi E, Giaccone G, Graeber MB, Ince P, Kamphorst W, King A, Korkolopoulou P, Kovacs GG, Larionov S, Meyronet D, Monoranu C, Parchi P, Patsouris E, Roggendorf W, Seilhean D, Tagliavini F, Stadelmann C, Streichenberger N, Thal DR, Wharton SB, Kretzschmar H: Staging of neurofibrillary pathology in Alzheimer's disease: a study of the BrainNet Europe Consortium. Brain Pathol 2008;18:484-496.

25 Braak H, Alafuzoff I, Arzberger T, Kretzschmar H, Del Tredici K: Staging of Alzheimer disease-associated neurofibrillary pathology using paraffin sections and immunocytochemistry. Acta Neuropathol 2006;112: 389-404.

26 Saito Y, Ruberu NN, Sawabe M, Arai T, Tanaka N, Kakuta Y, Yamanouchi H, Murayama S: Staging of argyrophilic grains: an age-associated tauopathy. J Neuropathol Exp Neurol 2004;63:911-918.

27 Kovacs GG, Budka H: Current concepts of neuropathological diagnostics in practice: neurodegenerative diseases. Clin Neuropathol 2010;29:271-288.

28 Montine TJ, Phelps CH, Beach TG, Bigio EH, Cairns NJ, Dickson DW, Duyckaerts C, Frosch MP, Masliah E, Mirra SS, Nelson PT, Schneider JA, Thal DR, Trojanowski JQ, Vinters HV, Hyman BT: National Institute on AgingAlzheimer's Association guidelines for the neuropathologic assessment of Alzheimer's disease: a practical approach. Acta Neuropathol 2012;123:1-11.

29 Williams DR, Holton JL, Strand C, Pittman A, de Silva R, Lees AJ, Revesz T: Pathological tau burden and distribution distinguishes progressive supranuclear palsy-parkinsonism from Richardson's syndrome. Brain 2007; 130:1566-1576.

30 Braak E, Braak H, Mandelkow EM: A sequence of cytoskeleton changes related to the formation of neurofibrillary tangles and neuropil threads. Acta Neuropathol 1994;87:554-567.

31 Insausti R: Hippocampal formation; in Mai J (ed): The Human Nervous System. San Diego, Elsevier, 2012, pp 896-942. 
Milenkovic et al.: Patterns of Hippocampal Tau Pathology Differentiate

Neurodegenerative Dementias

32 Arnold SE, Toledo JB, Appleby DH, Xie SX, Wang LS, Baek Y, Wolk DA, Lee EB, Miller BL, Lee VM, Trojanowski JQ: A comparative survey of the topographical distribution of signature molecular lesions in major neurodegenerative diseases. J Comp Neurol 2013;521:4339-4355.

33 Rabano A, Rodal I, Cuadros R, Calero M, Hernandez F, Avila J: Argyrophylic grain pathology as a natural model of tau propagation. J Alzheimers Dis 2014;40(Suppl 1):S123-S133.

34 Parvizi J, Van Hoesen GW, Damasio A: The selective vulnerability of brainstem nuclei to Alzheimer's disease. Ann Neurol 2001;49:53-66.

35 Thal DR, Holzer M, Rub U, Waldmann G, Gunzel S, Zedlick D, Schober R: Alzheimer-related tau-pathology in the perforant path target zone and in the hippocampal stratum oriens and radiatum correlates with onset and degree of dementia. Exp Neurol 2000;163:98-110.

36 Ferrer I, Lopez-Gonzalez I, Carmona M, Arregui L, Dalfo E, Torrejon-Escribano B, Diehl R, Kovacs GG: Glial and neuronal tau pathology in tauopathies: characterization of disease-specific phenotypes and tau pathology progression. J Neuropathol Exp Neurol 2014;73:81-97.

37 Luna-Munoz J, Chavez-Macias L, Garcia-Sierra F, Mena R: Earliest stages of tau conformational changes are related to the appearance of a sequence of specific phospho-dependent tau epitopes in Alzheimer's disease. J Alzheimers Dis 2007;12:365-375.

38 Puig B, Rey MJ, Ferrer I: Individual and regional variations of phospho-tau species in progressive supranuclear palsy. Acta Neuropathol 2005;110:261-268.

39 Guillozet-Bongaarts AL, Glajch KE, Libson EG, Cahill ME, Bigio E, Berry RW, Binder LI: Phosphorylation and cleavage of tau in non-AD tauopathies. Acta Neuropathol 2007;113:513-520.

40 Gilbert PE, Kesner RP, Lee I: Dissociating hippocampal subregions: double dissociation between dentate gyrus and CA1. Hippocampus 2001;11:626-636.

41 Kesner RP, Gilbert PE, Wallenstein GV: Testing neural network models of memory with behavioral experiments. Curr Opin Neurobiol 2000;10:260-265.

42 Kesner RP, Hopkins RO: Mnemonic functions of the hippocampus: a comparison between animals and humans Biol Psychol 2006;73:3-18.

43 Hyman BT, Van Hoesen GW, Damasio AR, Barnes CL: Alzheimer's disease: cell-specific pathology isolates the hippocampal formation. Science 1984;225:1168-1170.

44 Josephs KA, Whitwell JL, Parisi JE, Knopman DS, Boeve BF, Geda YE, Jack CR Jr, Petersen RC, Dickson DW: Argyrophilic grains: a distinct disease or an additive pathology? Neurobiol Aging 2008;29:566-573.

45 Dickson DW, Bergeron C, Chin SS, Duyckaerts C, Horoupian D, Ikeda K, Jellinger K, Lantos PL, Lippa CF, Mirra SS, Tabaton M, Vonsattel JP, Wakabayashi K, Litvan I: Office of rare diseases neuropathologic criteria for corticobasal degeneration. J Neuropathol Exp Neurol 2002;61:935-946.

46 Williams DR, de Silva R, Paviour DC, Pittman A, Watt HC, Kilford L, Holton JL, Revesz T, Lees AJ: Characteristics of two distinct clinical phenotypes in pathologically proven progressive supranuclear palsy: Richardson's syndrome and PSP-parkinsonism. Brain 2005;128:1247-1258.

47 Armstrong RA, Cairns NJ, Lantos PL: A quantitative study of the pathological lesions in the neocortex and hippocampus of twelve patients with corticobasal degeneration. Exp Neurol 2000;163:348-356.

48 Armstrong RA, Lantos PL, Cairns NJ: Hippocampal pathology in progressive supranuclear palsy (PSP): a quantitative study of 8 cases. Clin Neuropathol 2009;28:46-53.

49 Togo T, Cookson N, Dickson DW: Argyrophilic grain disease: neuropathology, frequency in a dementia brain bank and lack of relationship with apolipoprotein E. Brain Pathol 2002;12:45-52.

50 Jinno S, Kosaka T: Cellular architecture of the mouse hippocampus: a quantitative aspect of chemically defined GABAergic neurons with stereology. Neurosci Res 2006;56:229-245.

51 Freund TF, Buzsaki G: Interneurons of the hippocampus. Hippocampus 1996;6:347-470.

52 Klausberger T, Somogyi P: Neuronal diversity and temporal dynamics: the unity of hippocampal circuit operations. Science 2008;321:53-57.

53 McCall MA, Gregg RG, Behringer RR, Brenner M, Delaney CL, Galbreath EJ, Zhang CL, Pearce RA, Chiu SY, Messing A: Targeted deletion in astrocyte intermediate filament (GFAP) alters neuronal physiology. Proc Natl Acad Sci USA 1996;93:6361-6366.

54 Bezzi P, Vesce S, Panzarasa P, Volterra A: Astrocytes as active participants of glutamatergic function and regulators of its homeostasis. Adv Exp Med Biol 1999;468:69-80.

55 Bezzi P, Domercq M, Vesce S, Volterra A: Neuron-astrocyte cross-talk during synaptic transmission: physiological and neuropathological implications. Prog Brain Res 2001;132:255-265.

56 Agulhon C, Fiacco TA, McCarthy KD: Hippocampal short- and long-term plasticity are not modulated by astrocyte $\mathrm{Ca}^{2+}$ signaling. Science 2010;327:1250-1254.

57 Yang Y, Ge W, Chen Y, Zhang Z, Shen W, Wu C, Poo M, Duan S: Contribution of astrocytes to hippocampal longterm potentiation through release of D-serine. Proc Natl Acad Sci USA 2003;100:15194-15199.

58 Perea G, Navarrete M, Araque A: Tripartite synapses: astrocytes process and control synaptic information. Trends Neurosci 2009;32:421-431.

59 Bezzi P, Volterra A: Astrocytes: powering memory. Cell 2011;144:644-645.

60 Suzuki A, Stern SA, Bozdagi O, Huntley GW, Walker RH, Magistretti PJ, Alberini CM: Astrocyte-neuron lactate transport is required for long-term memory formation. Cell 2011;144:810-823. 